\title{
Internet community forums: An untapped resource for consumer marketers
}

Article in Journal of Consumer Marketing · August 2005

DOI: $10.1108 / 07363760510611699$

CITATIONS

80

2 authors:

\section{Dennis A. Pitta}

University of Baltimore

88 PUBLICATIONS 1,290 CITATIONS

SEE PROFILE
READS

361

Some of the authors of this publication are also working on these related projects:

Linking mathematically formal with socio-organisational Requirements Engineering techniques for high 


\title{
Internet community forums: an untapped resource for consumer marketers
}

\author{
Dennis A. Pitta and Danielle Fowler \\ University of Baltimore, Baltimore, Maryland, USA
}

\begin{abstract}
Purpose - To explore an emerging area in internet practice that has implication for consumer marketers. Design/methodology/approach - The paper integrates concepts including a range of recently published (1993-2004) theoretical works and ongoing case developments in internet practice.

Findings - Provides information and action approaches to consumer marketers that may increase the success, providing want-satisfying market offerings. Outlines the market research benefits of monitoring and participating in internet community forums and offers practical suggestions for maximizing their value in the marketing and marketing research. It also provides a series of tactics that consumer marketers may use to maximize the value of internet community forums for their firms.

Research limitations/implications - The theoretical concepts that form the foundation of the paper appear to have a significant application to consumer marketing, but have not been tested empirically.

Practical implications - Uncovers a previously unrecognized source of direct consumer input and cooperation in the design and valuation of new products and the identification of emerging consumer wants.

Originality/value - This paper describes the nature and application of internet community forums to an important marketing process. It offers the potential of increasing marketing success by clearly and accurately identifying the wants of specific market segments.
\end{abstract}

Keywords Internet, Consumer marketing, Communications

Paper type Conceptual paper

An executive summary for managers and executive readers can be found at the end of this article.

\section{Introduction: advantages of internet communication}

The internet, and specifically the world wide web, is acknowledged as a truly global means of connecting individuals and organizations. The characteristics of the internet foster its use among consumers who value convenience: asynchronicity, persistence, near instantaneous communications, specificity, and accessibility. Online forums are built on these advantages, while also providing a structure for the communications that take place.

Asynchronous communications allow one consumer to post a message to a forum hours, perhaps months, before another has a chance to see and respond to it. This asynchronicity frees consumers from a rigid communication schedule that would be difficult to meet and would suppress the numbers of people communicating.

Most internet web sites have an archive facility that stores visitor comments for review or retrieval. That feature provides a measure of persistence that can help consumers who visit

The Emerald Research Register for this journal is available at www.emeraldinsight.com/researchregister

The current issue and full text archive of this journal is available at www.emeraldinsight.com/0736-3761.htm

Journal of Consumer Marketing

22/5 (2005) 265-274

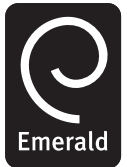

(c) Emerald Group Publishing Limited [ISSN 0736-3761]

[DOI 10.1108/07363760510611699] the site weeks or months after the comment was posted. Forums usually are divided into topic areas, with different topic "threads" running in each area. These threads may run for years; such archiving allows newcomers to access the "institutional memory" of the web site and its communications, so to learn its knowledge base and history.

Electronic communication is by its nature almost instantaneous and its speed aids discussion. While the structure of the public internet causes slight inherent delays, in comparison to traditional postal mail, they are insignificant. It is not uncommon for users to be online simultaneously and to have a computer mediated conversation, in the form of continuous posts and replies that are equivalent to an ongoing e-mail exchange. While even video streaming is possible at low-to-medium quality for many users, most forums are textmessage based, which is fast to compose and to post.

Specificity refers to the tendency of groups with similar interests to communicate online within virtual communities. These online communities are essentially market segments whose interests are very similar and much more specific than the population at large. Rothearmel and Sugiyama (2001) expressed concern for the effect of size on virtual communities. They found evidence of an optimal size for economic gain and effectiveness for members. That size relationship is curvilinear with very small and very large communities being less effective and productive than those of intermediate size. As most online forums have topic areas of interest divided into their own sub-topic areas, while the forum might as a whole grow into a very large size, its effectiveness for each of those smaller groups remains steady as long as they do not grow too large.

As information technology and the internet infrastructure have developed, accessibility has increased. Consumers can access the internet from home, work or other points ranging 
from public libraries to private sector internet cafes. In the developed world, those points of entry have proliferated over the last ten years. Today it is not uncommon to see users in open public areas accessing the world wide web on their laptops using wireless internet cards. The result is a large increase in communication volume and traffic, because consumers have the means to participate when it suits their lifestyle. Text-based forums are perhaps the most accessible. The reason rests in the so-called "last-mile problem" which refers to users' connection to their internet service providers. The link is the slowest link in the communication chain and is much less of a problem with text communication than with video or audio communication.

\section{Online communities}

Internet spending is projected to continue its growth well into the coming decade. Forrester Research estimates that by 2010 , online sales will reach $\$ 331$ billion. The company cites the growing population of online shopping households combined with retailer innovations and site improvements as the major drivers that will push e-commerce to 13 percent of total retail sales, up from approximately 7 percent in 2004 . Historically, online retailing has been primarily business-toconsumer (B-to-C). More recently, consumer-to-consumer (C-to-C) e-commerce has begun to show growth. Online auction sites such as eBay are perhaps the most well known instances of the C-to-C phenomenon; eBay's estimated revenue for 2004 was US $\$ 3$ billion.

As the frequency of online commerce is growing, so too is the degree to which consumers are using the internet to research purchasing decisions, including seeking out the online opinions of other consumers before they buy. A popular source of such opinion is an online forum devoted to the topic of interest in question. Rather than simply reading product reviews, a consumer can post specific information he or she is looking for, and question the authors of specific data on particular issues. Over time, as consumers become more familiar with the community members using the forum, they may establish relationships with some of them that extend outside the forum (often e-mail conversations, but possibly including meeting physically). In effect, the forum community is a C-to-C network.

The C-to-C relationship is an important one to marketers because relationships between consumers have been shown to influence brand choice (Wind, 1976) and the choice of services. Indeed, research has highlighted the importance of studying the relationships among communicators in the context of interpersonal communication networks (Reingen and Kernan, 1986). Understanding a community and its purchasing characteristics is obviously desirable, yet many communities have traditionally been difficult to distinguish, or their preferences difficult to determine accurately.

Online forums support communities formed around a specific interest. The interest might be in a particular product or service (e.g. the iPod: www.ipodlounge.com), a category of related products (e.g. Home Theater: www.avsforum.com; or wristwatches: www.pmwf.com), or a hobby or interest that involves a variety of products (e.g. hiking: www.azwilderness. com). Using sports as an example of a popular area, a forum can span the continuum from spectator sports to participative activities like fishing, skiing, golf and many others. Other common interest areas include metalworking, woodworking, travel, financial investment, automobile modification and repair, and electronics. In these forums, individuals share their thoughts and gain the insight of other knowledgeable persons. The specificity is remarkable. For example, a fishing forum area might be divided into the variety of fishing subtopics like deep sea, fly casting, fresh water, game fishing and others. It might even specialize on a specific fish species, like bass, trout or marlin. In one-to-one marketing terms, the contact is direct and highly interactive. Over time, as people learn more about each other and the subject, deep personal relationships may form.

The forum is the formal mechanism the community uses to exchange information, generally through posted messages that are organized into "threads", which are subject headers for a discussion. These threads are typically active for a while, with new postings being made, until the forum members lose interest in the discussion. At any time however, members may come across the thread and find they have something to contribute. In this way information is added over time, often with bursts of activity followed by periods of inactivity. These bursts are often predictable - such as following the release of a new product version, or a significant event. More importantly, the information is archived within the forum, giving both members and internet surfers searching for information access to the postings. New people discovering the forum are potentially influenced by what they read. If the information or advice is sound, and their interest continues, the consumer comes to rely on the forum and perhaps to actively contribute. As the forum readership grows it becomes more influential. Audioasylum.com and audiogon.com, for instance (both home audio forums, although audiogon.com emphasizes its marketplace) are now so influential that consumer audio magazines and manufacturers alike routinely mention them.

One of the benefits of online forums to marketers is that a range of consumer marketing data can be gathered on such communities, such as:

- data on satisfaction with a service or product within different targeted communities;

- data on desired, or undesirable, features for future products;

- data on degrees of brand loyalty, price sensitivity, etc. with different communities;

- data on unexpected markets or uses for a product;

- data on perceived direct competitors for different products; and

- changes in consumer attitudes over time.

Instead of using a variety of surrogate measures (age, income, geographic location, profession, etc., all indirectly aimed at identifying preference), or small representative samples (e.g. focus groups), online forums offer marketers the opportunity to observe a community of interest directly: their expressed preferences, satisfaction or dissatisfaction, and use of products or services they have in common. They also eliminate the bias of interviews or focus groups where the users are dealing directly with the marketer or their representatives.

\section{The nature of online communication: influential or superficial?}

Virtual communities are quite different than the real world. In traditional communities, people meet others first, get to know 
them and then, if the chemistry is right, form relationships. In the virtual world, people get to know others, form relationships and then, if the chemistry is right, choose to meet them (Rheingold, 1993). There is a more fundamental difference between a real and virtual community: communication mode. In the real world, much of the communication is face-to-face; in the virtual world it is not.

The term "computer-mediated communication" (CMC) refers to a type of interpersonal communication which operates in computer-mediated environments (CMEs) and is limited to a large extent to the printed word. In some cases images can carry some of the information transmission burden. In contrast, face-to-face communication describes an interpersonal communication setting in which all communicators share a common space and time context and can engage in communication where they can use all five senses.

CMC is growing in importance along with CMEs and the growth is expected to continue in response to government initiatives and the growth of e-commerce.

Online communication mostly follows the "cues filtered out approach" (Jettmar and Rapp, 1996). This refers to the stripping away of all non-verbal clues. The effect is due to the bandwidth of the CME and is often referred to as a "lean" medium. In face-to-face situations, individuals can reasonably see, smell, and hear others even if they are strangers. Many of these cues lead to conditions ranging from "love at first sight" to "immediate repulsion". It is difficult to minimize the cues' importance. In extreme conditions, sales people and confidence men can manipulate the non-verbal cues they present to boost their credibility.

Several communication theories have been applied to understanding the communication process underlying online communities. Two theories, social presence theory (Short et al., 1976) and media richness theory, (Draft et al., 1987) were used to study communication without non-verbals. Media richness theory stressed that different media have different capacities for supplying cues. Another approach, social presence theory, dealt with the feeling of being "present" in an environment. When most of the cues are not carried by the medium, the feeling of presence is low. The communication literature highlighted CMC's limitations. Empirical studies found it to be more task-oriented, less emotional, and less personal (Hiltz et al., 1986) than face-toface communication. It is possible that these findings might be due to the inability to transmit nonverbal cues.

There is contradictory information supporting a more robust form of online processing. The social information processing view holds that other mechanisms can compensate for the cue stripping effect of the narrow bandwidth bottleneck. Other factors such as impression development (Walther, 1994) may affect how we utilize CMC and its effects. The theory holds that the critical difference between face-to-face and CMC information processing is in the rate of impression development rather than the mode's capacity.

Since online communications are restricted to one sense rather than all five, they offer fewer impression development cues per time unit. However, over time, CMC can deliver enough to help form relationships. In addition, online communicators were found to use "strategic probes" when decoding text-based cues. These strategies may foster developing impressions of the other person which are as accurate as those formed face-to-face.
Whether or not they are as rich and accurate as traditional face-to-face relationships, online communities offer perhaps the strongest online variation, as they develop slowly over time, based on multiple communications that have a common context (the topic of the forum).

\section{Utilizing online forums}

\section{Traditional one-way communication: marketer to consumer}

Recognizing the deleterious effects of negative word of mouth (WOM, the classic original C-to-C interaction)), numerous authors have investigated the effects of complaining behavior by consumers on company success (Charlett and Garland, 1995; Richins, 1983). In contrast, positive WOM may be the one measure of a company's prospects for success. Reicheld (1996) explored the evidence supporting a customer's brand loyalty. He found that the most important single factor that correlated with company growth was the willingness of customers to recommend a company or brand. When a customer recommends a company, he/she incurs certain risks. If a friend acts on the recommendation and tries a brand or company and is not satisfied, the source may lose credibility and suffer damage to his/her reputation. Reicheld predicted that consumers would only make such recommendations if they feel intense loyalty.

There is some research on C-to-C relationships in noncommercial environments in which the exchange of money for goods and services is not a primary basis for interaction. For example, research on the shared brand preferences between members of a sorority was found to arise from routine personal interaction rather than commercially based factors. Moreover, WOM information has also been linked to noncommercial sources (Richins, 1983).

Taking WOM as the most examined form of C-to-C marketing, the communication of information takes the form of one consumer talking to another about a product or service. Such direct interaction is typically limited in scope. However the WOM model allows for more complex communication, including groups of individuals sharing information. They include common interest groups such as book clubs. Still, the WOM process is limited by the need for direct communication.

Marketers, who exploit WOM, aim to make the discussion positive and they have honed the techniques of generating positive WOM. They have even integrated the concept into a two-stage advertising model in which they advertise to opinion leaders (not role models but experts you know) who in turn tell their friends (see Figure 1).

While the marketer may set things in motion, he/she is not part of the C-to-C interaction and may not know the results. Online forums give marketers the opportunity to see these results, although care must be taken when interacting with them actively, as we will explain shortly.

One-way communication from consumer to marketer In viewing the internet, its communication facilitating aspect is critical. The internet can foster interaction characterized as: one-to-one, one-to-many, many-to-many, and even many-toone. When the internet was in its introductory stage, marketers studied it as a potential new marketing medium (Hoffman and Novak, 1997). It was at that time that the internet was called a CME. Computers, linked in a virtual 
Figure 1

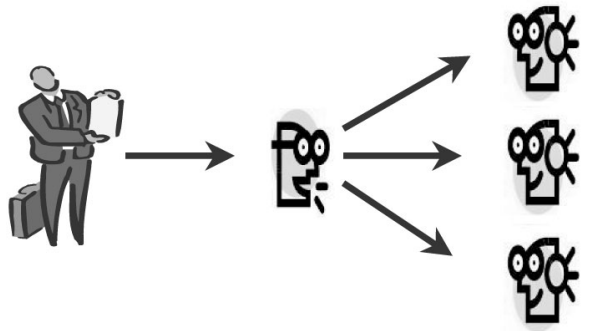

Advertiser Opinion Leader Consumers

network, comprise the backbone of the world wide web. Consumers who interact on the web use those computers as one of their communication tools. Thus the structure of the network and the nature and capabilities of computers constrain consumer interaction. From the beginning, it was clearly a valuable communication tool and logically, organizations searched for a means of using the new medium for marketing. Initially, what developed was a oneto-many communication device. In the typical model, an organization sent information content to a web site that served to transmit it to many target customers (Figure 2). The communication is typically static, press-release type information that the browser pulls from the company's own site, or advertising based (e.g. banner advertisements) on other sites, and the channel is one-way.

Companies still use this static "pull" model in their web sites. Individuals can visit the sites for material ranging from warranty information and product manuals to press releases. While the model may be an efficient means of contacting multiple consumers, its one-way nature limits effectiveness. Consequently, most organizational web sites also now incorporate an e-mail-based consumer contact function.

\section{Two-way communication: $\mathrm{C}-\mathrm{C}$, and consumer-}

\section{marketer}

Discussion forums present a different $\mathrm{CME}$, one with greater interactivity and interpersonal use. In this case, a consumer can post a message to a forum and wait for others to react. The simplest model shows two consumers interacting by each sending their message to a forum (Figure 3).

A message which is sent by the first consumer to the forum can be read by another. The other consumer can send his/her

Figure $\mathbf{2}$ The static one to many marketing communications model

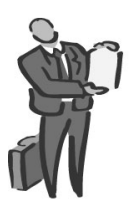

Firm

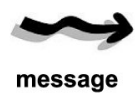

message
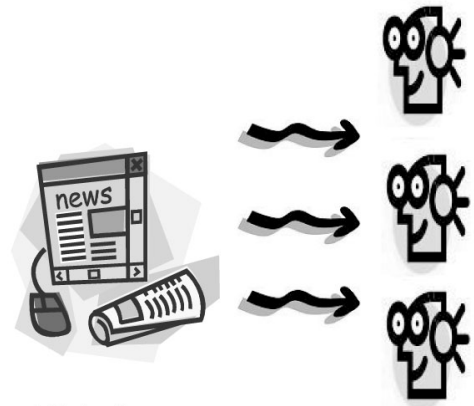

Consumers
Figure 3 Interpersonal computer-mediated communication

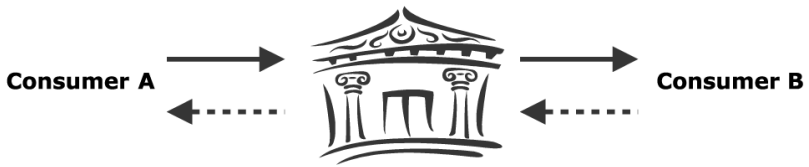

own return message to the forum where the first consumer can see it. The mediated nature of the communication has benefits and drawbacks mentioned above. Its interaction is far from dyadic. In fact, multiple consumers can read each message. Thus, the forum design supports two-way, many-tomany communication. The receivers can be consumers or other entities like firms. Thus each of the individuals listed in the model can interact through the hub represented by the forum.

There is one communication link not depicted in Figure 4: direct C-to-C interaction. Most discussion forums provide either a private messaging function or will provide a particular member's e-mail address. Consequently, forum participants can communicate with each other in private.

It is unquantifiable, but much C-to-C communication in discussion forums seems to take place outside the public forum. Most forums provide a private messaging function, which allows individuals to interact directly, shielded from public view. For individuals concerned about their privacy, private messages sent within the framework of the forum can request e-mail addresses. Those addresses will permit individuals to interact free from the fear that a moderator or other members may see their messages. The effect of such private communication is to build relationships between individuals.

In fact this offline communication may represent a maturing of the online community resulting in firm friendships among some of the members. That friendship may be expressed by direct electronic or telephonic communication. It also takes the form of face-to-face meetings at events or visits.

This multipath communication gave rise to the notion of the internet as a community of members interacting among themselves. In fact, that notion has been central to the internet from its inception (Hagel and Armstrong, 1997).

Marketers do not usually adopt a "community" view of their market. They tend to focus on the wants and characteristics of their target market segments. Segmentation efforts by marketers have undeniably been successful over time and have developed into an effective tool in the quest to satisfy consumers. Still much of the

Figure 4 Extended computer-mediated communication

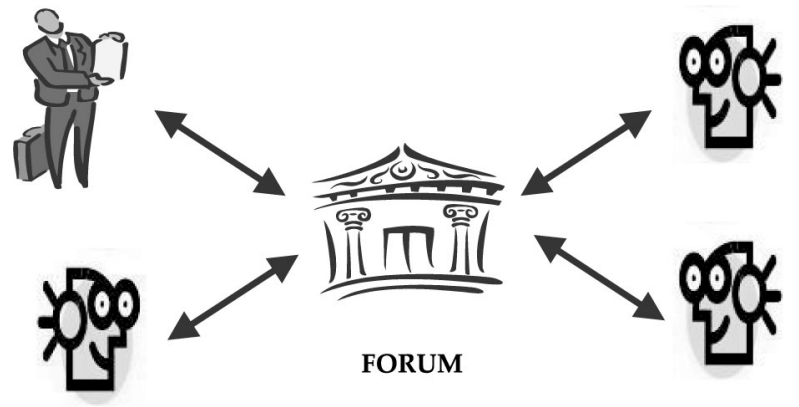


segmentation effort is one way. Marketers try to gauge consumer tastes using various research techniques, then aim promotions at their target segments. While marketers may monitor consumer's reactions, the information flow is far less and of lower quality than the "open talk among friends" generates.

In contrast to marketing, the view of customers as part of a community is central to anthropological research. That research tradition holds that there are preeminent elements defining communities. Historically, they have included:

- sustained social interaction;

- shared attributes, interests and values (community standards); and

- a proscribed geographical area.

Those elements define cities, towns and villages throughout the world. The definition is limited, however, and does not include the numerous other communities that exist. For example, university alumni associations and veterans groups may have members scattered around the globe. They can interact via telephone, mail, or e-mail and can even meet for special occasions.

The internet presents its own geography, which has no traditional geographic boundaries. As a result, more modern research has accommodated the boundless confines of the internet in its definitions. For our purposes, a community exhibits: sustained social interaction; shared attributes, interests and values (community standards); and membership rules. These elements are present in "virtual communities", those groups of interacting individuals which form on the internet. The focus of each community may be technical, social, and economic or a special interest shared among members. These online virtual communities allow that necessary social interaction among members and have either subtle or conspicuous rules of behavior.

Interaction among the members is the engine that fosters the growth of such communities. That interaction is part of a relationship building process that allows individuals with common, and perhaps rare interests, to communicate together in pursuit of those interests (Hagel and Armstrong, 1997). The communication process takes place in a CME that shapes the nature and scope of the information shared. CMEs have some strengths that aid communication effectiveness as well as weaknesses that hinder it. One strength is the use of discussion forums that allow asynchronous communication. The forums' asynchronous nature fosters communication because it avoids having to schedule a time when comments are shared. A user can post comments; others can respond to them later.

In contrast, CMEs are limited to a subset of communication modes. Until recently, one could use text and graphics, but sound, smell and body language could not be shared. Even now, embedding sound or video clips is ponderous and not available to all. One other drawback is that its asynchronous nature sterilizes spontaneity.

On the other hand, such CMC allows time for reflection and thought that can increase communication effectiveness. In addition, careful crafting of messages or a response to a message can avoid unintended insult or confusion. The asynchronicity inherent in CMEs allows communicators to verify their statements, bolster them with proof or other information and helps avoid falling into a useless exchange of opinions not grounded in fact.
A virtual community depends on combining communication and content to foster the exchange of information. By its nature, the information exchange allows people to learn about each other as they learn more about the community's focal topics. Hagel and Armstrong (1997) have investigated the consumer motivations for joining an online community. They satisfy several needs. Three are of relevance here:

(1) shared interest;

(2) relationship building; and

(3) transaction.

Online communities can satisfy all three needs in equal measure or concentrate more on one or another. As Rothaermel and Sugiayama (2001) point out, this can lead to several types of communities. A community of interest comprises individuals who share a common interest, hobby or skill set. In a community of interest members will share their experiences, preferences and information about the focal topic. For example, bass fishermen may discuss lures, bait, fishing grounds and gear. The second element, relationship, can lead to a community of relationship. These form around some intense life experience such as the loss of a loved one, a disease diagnosis or other significant event. In such circumstances, consumers may find comfort in the experiences of others and, in doing so, form relationships with them. Over time the relationships become increasingly important. Finally, a community of exchange focuses on the exchange of information to facilitate economic exchange. Thus, audiophiles can discuss the newest releases, and innovative equipment and the best sales sources in a given area. In each of these communities, members share information of value to themselves and others. That sharing can be valuable to marketers.

\section{Operation of online communities}

The typical online community is a niche. The interest relationship or exchange focus is usually very narrow. Logically, online communities satisfy needs not met in faceto-face situations. A member's real local community may have a large population with few or no people interested in the same subject. If the focal issue is narrow, the online community will mirror that narrowness and tend toward homogeneity. As a source of market information such online communities can be priceless.

A discussion forum is a unique blend of public and private communication. They make the risk of participating lower. They force individuals who want to participate to register online at no cost, reducing the monetary risk of joining. Registration involves supplying identity and contact information as well as a preferred nickname to preserve anonymity in discussions. Typically, users are asked for a valid e-mail address, and possibly an address. When supplied, a registration password is returned to the user's specified e-mail address. This practice serves as a partial safeguard against fraudulent behavior, reducing that risk. Without a valid e-mail address, a user cannot register. Even if the contact information is false, the e-mail address will aid in identifying the user should a problem arise.

The use of pseudonyms has another benefit. They allow members to recognize the postings of others and to foster a sense of a member's personality. Over time, members form 
impressions of others, which may reflect their interests, knowledge level, and even the quality of their judgments and their character. In many respects, that online persona serves the same function as a brand and allows a person to establish his or her own brand identity.

\section{Relationships}

Members may find an online community after an internet search. Their initial interaction may be as a "lurker". Lurkers visit a discussion forum and may initially read the posts and observe without participating actively. The reaction is understandable since learning the group's mores and expectations is important. As individual personalities emerge, new members may become comfortable enough to participate themselves and share their own preferences and interests. Protected by the anonymity that a pseudonym provides, members may divulge information in a public forum that few others may know.

The information sharing process is at the heart of a consumer-to-consumer network. The key obstacle to sharing is trust. The use of pseudonyms and the public sharing in the discussion tends to build trust over time.

The relationship-building aspect of online communities is the basis for consumer-to-consumer networks. The process develops in the following way. Consumers interact in a discussion board focusing on issues of interest. If the community rules provide no barriers to participation, a group of members may start interacting, discussing topics of mutual interest. Over time, other members will read the posts and associate them with their authors. By linking authors to their opinions, judgments and words, members can build an image of other online members.

During the interaction process the members, identified by their screen names, may post comments directed at a specific member. They may be complimentary or solicitous and, if positive, they tend to build relationships. Using the private messaging facilities, members may contact each other and form their own mini-network of members. Over time the relationships can become strong and members may even travel to meet and socialize.

The information network offers a rich and unparalleled source of information and ideas for marketers. To exploit this source, marketers may wish to use their own pseudonyms and visit the forum to observe its content. By shadowing the forum, marketing personnel can gauge the nature and scope of the interaction that might reveal consumer complaints, suggestions, wish lists, or new ideas. The arena allows an open window to the consumer's expressed opinions, beliefs and preferences. Over time, consumers learn to trust the safety of their group of online friends and divulge more deeply. They witness others sharing information and tend to join in. While it is difficult to estimate the value of this information source it must be considerable.

Borrowing from the new product development literature, such forums foster the emergence of "lead users" (von Hippel, 1986). Lead users are very knowledgeable about a topic area and often anticipate new product applications or modifications. They have provided a great deal of information to aid the new product development process. Their expertise may make them opinion leaders for their peers. If marketers are able to see the community's communication, there may be ethical concerns. Those concerns can be reduced if members know that their comments are public and open for all to see.

\section{The full continuum of marketing information}

Discussion forums present an unprecedented opportunity for both passive market intelligence and active market research. Listening to the dialog can alert companies to possible future trends, an example of market intelligence. Verification might require active marketing research. Here the marketer can be more than observer and reporter. Researchers can enter the forum and conduct discrete, focused surveys after determining the characteristics of the members. Beyond survey research, a discussion forum may provide a venue for experimentation. For example, creating "membership-only" offers for a discussion forum is a form of field test that might provide metrics of consumer price elasticity, preference for product features and other valuable information.

\section{Sponsorship}

Marketers can design, foster, and implement such C-to-C networks for a generalized, mutually beneficial exchange relationship. In traditional broadcasting sponsorship, the aim is to promote the brand or company image. That goal is accomplished by aiming commercial messages at target consumers. The internet easily adopted sponsorship in the form of pop-up or banner advertising. Integrating IT functionality, online sponsorship allows "click and view" exposure to advertising. Despite the emergence of pop-up advertisement "killers", such advertising exposure is cheap and offers the potential of delivering a group of self-selected prospects. One fundamental problem with all advertising is its one-way nature. Advertisers can beam messages at an audience but have difficulty gauging their effects, other than sales. If sales response is low, it might be due to the offer suiting only a narrow range of customers. Alternatively, it might be favored by most customers but be flawed by a single element. Perhaps the price is right but the payment terms are not. One-way communication thwarts really understanding the consumer.

Ignoring the promotional possibilities, IT and the internet offer a wider way to interact with consumers. Today, sponsorship entails designing, funding and operating an online forum. For example, Black and Decker, the power tool manufacturer, might sponsor a free discussion forum focusing on various woodworking topics. The discussions would probably attract numbers of woodworking enthusiasts, generate considerable information, and increase the knowledge level of the members.

What would Black and Decker receive for its investment? It would gain a cornucopia of detailed consumer wants along with the ability to clarify underlying details. The online discussion might point to the emergence of a new tool and how consumers actually use it. Additionally, it would serve as a source of competitive information since consumers would probably comment on tools in general and some competitor's tools specifically.

Extended sponsorship provides the arena for consumers to interact with other consumers. However, companies that do not understand the nature of online communities and the expectations of their members may make strategic missteps. 


\section{Online community rules of behavior}

Online discussion forums are subject to the same fashion trends as other gathering places for individuals. Places such as restaurants, hotels and bars can maintain their popularity by catering to customers and continuing to reinforce a feeling of welcome. The best physical gathering places seem to be less place than people. In other words, the reason for visiting is interacting with interesting people, not entering a familiar space. That observation also applies to online forums. Since one can leave a virtual place with speed and ease, online communities are vulnerable to problems caused by member behavior. A few "bad" members can kill a community.

In most cases, there is a sophisticated set of rules of expected behavior. In general, members expect to see information of interest and respectful behavior. If the quality of information declines, members can usually go elsewhere to meet their needs. Moreover, disrespectful behavior by members toward each other can lead to dreaded "flame wars", characterized by overt criticism and insult. Flame wars are a primary reason for discussion forum failure and the break up of online communities. When such behavior appears, there is a series of strategies used to contain it. They include:

- peer pressure;

- moderator pressure; and

- outright banning.

Peer pressure is often the first step in which other members ask the parties to restrain their comments. The second, more advanced, step is for a forum moderator to warn the offending members to cease and desist. The final step is to ban the offending member from posting on the forum. The actions are vital in preserving an open and valuable place for communication. There are other behavioral rules that are important, depending on discussion area.

Online discussion forums possess three main types of discussion areas: ordinary discussion forums; a "marketplace" forum in which items are bought, sold, traded or sought; and a forum paid for by a commercial sponsor. In some cases a single company that provides discussion areas for visitors can sponsor entire discussion sites. The sponsorship is overt and visitors expect some commercial character.

The rules for behavior are strict and specific to the particular discussion area. Behavior that is allowed in one area may be an offense in another. The matrix shown in Table I serves as a decision guide for individuals and organizations.

The matrix in Table $\mathrm{I}$ is divided into several categories based on communication mode and the area of the discussion forum. The three major areas are arranged horizontally. Four major communication modes are arranged on the vertical axis. They are, basically, active or passive, solicited or not solicited. Due to the characteristics of the internet they break down as active-solicited, active-unsolicited and passive. The last category accounts for the last major communication mode, a private offline communication to another member.

\section{Location of online discussion}

\section{Discussion forum}

The most sacrosanct area is the discussion forum, the area in which consumers discuss items of interest. There may be numerous discussion forums segmented according to the specific interests of the community. Thus, a travel-focused community may segment according to destination with major divisions such as Europe, Asia, and the rest of the continents. Further segments can be country or even city specific. The primary reason that consumers visit the forum is to interact with other consumers and get consumer-based information. They look for opinions, experiences and tips to help them. In the case of a travel-focused forum, members seek facts and recommendations from others they trust. In essence they are looking for word of mouth. Thus if one member asks another for recommendations of a hotel in Venice plus a list of restaurants and shopping sites, the entire forum benefits. If there is a hint of commercialism the perceived value of the information goes down. One member on lonelyplanet.com, a travel site, commented that he was tired of "maître de"-level information. He was referring to recommendations that hotel staff make to visitors that seem to be motivated not by the customer's needs, but the kickback the staff got. In contrast, he trusted the responses from the members.

Members expect non-commercial interaction in discussion forums. Marketers who wish to use discussion forums for commercial purposes should be aware of what consumers expect and tolerate, and what they dislike. One commercial interaction that is acceptable is a response to a consumer request for information. They take the form of asking for advice about where to find a particular product or service. Since a member requested the information, it is permissible for a sponsor or other commercial entity to answer that question. If, however, a member acting as a company agent or "shill" asked the question, members who are aware of the true identity of the other member may take offense. More overt intrusions are also likely to be annoying. The level of consumer tolerance for the postings from a manufacturer or company can be built slowly over time. If the focus of the contributions is on details the forum wants and cannot get

Table I Behavioral rules for online communities

\begin{tabular}{|c|c|c|c|}
\hline \multirow[b]{2}{*}{ Communication mode } & \multicolumn{3}{|c|}{ Location of online communication in the forum } \\
\hline & In the marketplace & In a discussion area & In a marketer sponsored area \\
\hline $\begin{array}{l}\text { ctively solicited } \\
\text { ommunication (postings) }\end{array}$ & $\begin{array}{l}\text { Accepted and effective behavior if } \\
\text { ethical }\end{array}$ & Accepted if post is on-point & Accepted - a source of marketing data \\
\hline $\begin{array}{l}\text { Unsolicited communication } \\
\text { (postings) }\end{array}$ & $\begin{array}{l}\text { Accepted and effective behavior if } \\
\text { ethical }\end{array}$ & $\begin{array}{l}\text { Negative - may be perceived as an } \\
\text { intrusion }\end{array}$ & $\begin{array}{l}\text { Accepted if the source is the sponsor } \\
\text { Negative if the source is a competitor }\end{array}$ \\
\hline $\begin{array}{l}\text { Passive communication } \\
\text { (read without posting) }\end{array}$ & $\begin{array}{l}\text { Positive effects - a potentially good } \\
\text { source of marketing information }\end{array}$ & $\begin{array}{l}\text { A good source of information about } \\
\text { consumer wants and needs }\end{array}$ & $\begin{array}{l}\text { Positive effects - a good source of } \\
\text { competitive information re the sponsor }\end{array}$ \\
\hline $\begin{array}{l}\text { rivate messages off the } \\
\text { liscussion forum }\end{array}$ & \multicolumn{3}{|c|}{$\begin{array}{l}\text { Private consumer to consumer messages are hidden from public view and may be the most valuable information } \\
\text { source for marketers }\end{array}$} \\
\hline
\end{tabular}


elsewhere (perhaps specifications of future products), then the posting is considered informative, and neutral. It is the individual poster who is considered an asset over time, however, this does not mean another from the same company automatically enjoys the same privilege. The important corollary here is that while trust is slow to build, it can be very quickly lost. Posting to the open discussion forum is therefore the most critical type of active interaction a marketer can engage in, and must be approached with care.

The last type of action in the discussion forum is not interaction at all. It is the passive monitoring of the flow of discussion and imperceptible. It is also an excellent source of accurate consumer information. Both consumers and marketers can benefit from this type of action, free of potential problems from other consumers or competitors.

\section{The marketplace forum}

Most online community web sites have an area reserved for buying, selling, trading and posting of want to buy messages. This may be divided into a trading post (used individual sales), marketplace (commercial sales), and a swapmeet (for trades, rather than sales). In the marketplace, anything goes. Individuals and companies can offer items for sale and the only expectation is that buyers and sellers will act ethically.

The marketplace forum may be an important profit center for marketers. Passive monitoring can provide valuable data. Armed with very specific consumer preference information and the daily experiment that is the marketplace, companies can structure deals and offers that are accurately designed and priced. There may be a cost to contacting an online community. Forum sponsors are in a profit-seeking venture and they may charge companies a membership or transaction fee for the use of the marketplace. Since the costs of contacting an online community are usually minimal, companies have the ability to reach a targeted audience with a highly refined offer with a good chance of profit.

\section{The marketer-sponsored forum}

It is important to distinguish between a marketer-sponsored discussion web site and a marketer-sponsored forum. If a marketing company sponsors an entire web site, its commercial purpose is overt. The sponsoring company usually will not welcome competitors. In contrast, third party web site owners usually welcome sponsors. To increase the perceived value of the site, such independent owners may encourage other companies to form their own sponsored discussion forums on the site, usually for a fee. Such actions increase the "one-stop information shopping" value that can attract more visitors and will boost overall revenue.

On web sites that serve as umbrellas for different retailers, competitors may have their own sponsored discussion forums. The sponsored forums may deal with customer service issues, requests for marketing research or consumer preference information, or highlight special offers restricted to members.

In several sites surveyed, the web site managers have strict rules of behavior for their paid sponsors. The goal is to avoid the sniping and "flame wars" that can harm the entire web site. The rules tend to restrict active and overt competitive actions. Thus, sponsors are instructed to respect others' turf and not to trespass. There is usually a general gag order to avoid one competitor criticizing another. However, since the information and promotional offers posted in the sponsored forum are public, it is difficult to police "conscious parallelism", the duplication of specific promotional offers or new products. In the past, conscious parallelism referred to the practice of matching a competitor's price, without an overt agreement to fix prices at a higher than competitive level. In this case, conscious parallelism is an attempt to be more competitive by matching a low price.

While travel-focused sponsors can easily exploit this form of conscious parallelism, retailers who sell products on sponsored web sites can also use the technique.

\section{Offline communication}

Offline communication represents the most powerful yet immeasurable effect of online communities. When members build relationships that reach a level of trust that prompts a personal contact, they interact like friends sharing word of mouth. They can recruit others to share travel- or community-related purchases or other activities. In fact, offline communication among online "friends" appears to be one reaction to online problems with discussion forums. Recently, the owner of a sports-related web site with multiple discussion forums tried to institute a fee for membership in a particularly clumsy and insulting manner. As a result, seven members formed their own voluntary parallel web site. They recruited disaffected members of the original site and slowly built membership. When economics forced them to institute a fee for membership, they handled it in an effective way. This illustrates a problem with managing the web sites and the online community. Another possibility is that offline communication on a general forum results in the creation of a more specific forum, catering to the issues that prompted the offline communication.

In summary, if marketers follow the rules of behavior with the objectives of building trust, acting ethically, and treating members responsibly, they may avoid the threats that damage online communities and hopes of marketing advantage.

\section{Implications for marketers}

Marketers should consider seeking out and sponsoring online discussion forums featuring topics of interest to their customers. The involvement may take the form of sponsoring or hosting an entire forum, or a part of a forum. One key is early involvement with interested individuals with a passion for the subject. They are likely to attract others and form a community.

The growing numbers of people who use the internet present a growing audience for marketers. Sponsored online discussion forums convey valuable marketing benefits. They form an interactive window to an audience that can provide a conduit for the voice of the customer. Opportunities to interact deeply with consumers over the long haul are rare. Sponsored forums provide unprecedented interactivity. However, marketers must be mindful of the rules of the road. Violating customer expectations of behavior can tarnish a company's image and damage a sponsored forum.

The literature and practice show that online communities can change the marketing research function. The forums can be a source of exploratory research, concept testing, product design, delivery preferences, communication research, and even pricing. Since online forums cater to very specific needs, they seem to attract somewhat homogeneous membership. Thus they can convey an audience with low within group differences that can represent specific market segments well. 
The depth of resulting information can be extraordinary and be of extraordinary value.

Online communities can also change the marketingcustomer relationship. An online discussion board can become a source of expertise that turns it into a specialty product. Getting the information and interacting with the other members becomes more important than price. Sponsors of popular discussion forums may be able to mold customer expectations about quality and price and exploit what amounts to a captive market. Online communities offer the potential of an unprecedented link between marketer and consumer that may yield a robust relationship. If marketers can provide expert information for specific communities, they can gain an expert position and position of trust. Achieving such a position is a point of differentiation and can translate into economic success.

Knowing the results of a horserace before it is run is the elusive goal of gamblers and gangsters. In effect, astute marketers who understand online communities can gain that type of prescient information. Knowing what the consumer wants before products or offers are created increases the efficiency of the marketing transaction and its ultimate effectiveness. It can provide an unassailable competitive advantage.

In order to exploit the online community information source, organizational changes may be necessary. Companies will need to assign internal personnel to monitor or perhaps lead discussion threads. The information flow they generate may force the creation of a new marketing information system in which passive marketing intelligence from discussion forums is channeled to decision makers who may require more active marketing research. Failure to organize the information management effort threatens to waste what might otherwise be an enterprise boosting resource.

\section{References}

Charlett, D. and Garland, R. (1995), "How damaging is negative word of mouth?", Marketing Bulletin, Vol. 6, May, pp. 42-51.

Draft, R.L., Lengel, R.H. and Trevino, L.K. (1987), "Message equivocality, media selection, and manager performance: implications for information systems", MIS Quarterly, Vol. 11, pp. 355-66.

Hagel, J. and Armstrong, A.G. (1997), Net Gain: Expanding Markets through Virtual Communities, Harvard Business School Press, Boston, MA.

Hiltz, S.R., Johnson, K. and Turoff, M. (1986), "Experiments in group decision making: communication process and outcome in face to face versus computerized conferences", Human Communication Research, Vol. 13, pp. 225-52.

Hoffman, D. and Novak, T. (1997), "A new marketing paradigm for electronic commerce", Information Society, Vol. 13 No. 1, January-March, pp. 43-55.

Jettmar, E. and Rapp, M.W. (1996), "Computer-mediated communication: a relational perspective", Pasadena, available at: www.danger-island.com/ true/papers/ CMS.html (accessed 15 August 2001).

Reichheld, F.F. (1996), The Loyalty Effect, Harvard Business School Press, Cambridge, MA.

Reingen, P.H. and Kernan, J.B. (1986), "Analysis of referral networks in marketing: methods and illustration", fournal of Marketing Research, Vol. XXIII, pp. 370-8.
Rheingold, H. (1993), The Virtual Community: Homesteading on the Electronic Frontier, Addison-Wesley, Reading, MA.

Richins, M. (1983), "Negative word of mouth by dissatisfied customers: a pilot study", fournal of Marketing, Vol. 47, Winter, pp. 68-78.

Rothearmel, F.T. and Sugiyama, S. (2001), "Virtual internet communities and commercial success: individual and community-level theory grounded in the atypical case of TimeZone.com", fournal of Management, Vol. 27, pp. 297-312.

Short, J., Williams, E. and Christie, B. (1976), The Social Psychology of Telecommunications, Wiley, London.

von Hippel, E. (1986), "Lead users: a source of novel product concepts”, Management Science, Vol. 32 No. 7, July.

Walther, J.B. (1994), “Anticipated ongoing interaction versus channel effects on relational communication in computer mediated interaction", Human Communication Research, Vol. 20, pp. 473-501.

Wind, J. (1976), "Preference of relevant users others in individual choice models", fournal of Consumer Research, Vol. 3, June, pp. 50-7.

\section{Further reading}

Grayson, K. (1999), "When consumers collide: consumerconsumer relationships in commercial environments", Advances in Consumer Research, Vol. 26, pp. 416-18.

Lawrence, T.B. (1995), "Power and resources in an organizational community", Academy of Management Best Papers Proceedings, pp. 251-5.

Martin, C.L. (1996), "Consumer-to-consumer relationships: satisfaction with other consumers' public behavior", The Fournal of Consumer Affairs, Vol. 30 No. 1, pp. 146-69.

Moon, Y. (2000), "Intimate exchanges: using computers to elicit self-disclosure from consumers", The fournal of Consumer Research, Vol. 26, March, pp. 323-39.

\section{Executive summary}

This executive summary has been provided to allow managers and executives a rapid appreciation of the content of this article. Those with a particular interest in the topic covered may then read the article in toto to take advantage of the more comprehensive description of the research undertaken and its results to get the full benefit of the material present.

\section{Online marketing - participant observation and real conversation}

During the first decade or so of internet usage and the world wide web, marketers thrashed around looking for ways to take advantage of this exciting and growing communications medium. At first we saw online communications as an advertising medium - another route to get our message in front of consumers. Advertising agencies began to buy space on web sites on which to put promotional messages for their clients.

The upshot of this explosion in paid for advertising was two-fold. First, consumers using the web got irritated by the ads, pop-ups and related intrusions into their smooth involvement in the online world, and second, the advertisements stopped working (which is perhaps related to the irritation levels). 
Running parallel to the activity of traditional advertisers was the engagement of direct marketers with the internet. These marketers, drawing on a tradition of consumer dialogue recognized what Pitta and Fowler also note - that the internet presents a real opportunity for "one-to-one" marketers and a real conversation with customers. Initially this work concentrated in the business-to-business world since businesses were first online and began to use the internet to deliver business benefits and efficiencies.

At the same time the research community began to see opportunities for their work. At first the internet was seen as a cost-effective means of questioning businesses and consumers. Following from this was applying the internet to intelligence gathering and information collection. But, just as with traditional and direct marketers, all we had done was to transfer existing methods from the real to the virtual world.

Pitta and Fowler begin to take us beyond this straight line thinking by examining the role of online communities and the ways in which they interact. This takes us into the experience of other communications disciplines - public relations and corporate communications. It is the world of that "buzz marketing" referred to by Bush et al. elsewhere in this edition of $\mathcal{F C M}$.

Anthropological research and online communities

Marketers have tended to study individuals and agglomerations of individuals using statistical techniques to establish norms and patterns within consumer behavior. While we have always recognized the existence of other research traditions - occasionally making use of them in our work - we remain wedded to number crunching. Social anthropologists apply a different approach to research by becoming participant observers within the community or society being studied.

Pitta and Fowler observe that the online world - just like the real world - is inhabited by distinct communities operating to established rules of behavior and having a specific set of rituals. The "geography" of these communities is different in that they are not, in the main, determined by physical proximity but by shared interests, relationships or activities. For the anthropologist (and the marketer applying anthropological methods of investigation) these communities are interesting since they will be typified by differences from other groups.

In general terms the marketer engages with these online communities by the process of becoming engaged - being a participant. We are able to observe the dynamics of the community, to appreciate the subtleties of the participants' language and to delimit the social and ethical mores that govern behavior within the community. And, unlike real world communities, these online groupings are brought together by a shared experience or interest rather than an act of geographical accident. This act of gathering together to share performs part of our marketing by creating identifiable clusters within our target markets. Becoming a participant not only allows us to gather useful product feedback and market intelligence, but also permits (within the rules of the community) a real conversation with consumers.

Watch your language

The social anthropology discipline is full of stories of researchers who have caused offence and upset by stepping beyond the bounds of acceptable behavior. The same goes for commercial engagement with online communities where, very often, blatant commercialism is frowned on by members of the group. Similarly, direct criticism of competitors or competitor products is not welcome.

Pitta and Fowler provide a helpful table setting out the types of communication that are acceptable within different communities and under different circumstances. It is safe to say that most of these group welcome information from businesses providing products or services of interest to members. But there is a resistance to the hard sell and a preference for the provision of information that assists in choice.

The good online marketer will establish a presence in the specialist community, allowing misconceptions to be corrected, information to be provided and genuine experience to be collected. All this assists in the design of products, the setting of prices and the delivery of customer service. We should aim to be seen as a friend online, playing a part in the life of the particular community. Pitta and Fowler are right to comment that our membership is specific to an individual rather than to the business itself - we need to ensure that our presence within the community is not compromised by employees who move on. At the same time we should make sure that, with more than one employee engaged within the community, we maintain consistency of message.

Online marketing has moved on as we recognize that the internet is creating a new set of behaviors. These are adaptations of behaviors from the real world but are played out in the particular environment that exists online. As businesses we are very vulnerable to attack from these online communities, but equally have the opportunity to develop effective marketing strategies using a genuine conversation with actual and prospective customers online. What is important is that we retain a sense that this is discourse rather than a sales monologue. The online world has become more sophisticated and, while welcoming information, guidance and advice, rejects the overt commercialization of online activity.

(A précis of the article "Internet community forums: an untapped resource for consumer marketers". Supplied by Marketing Consultants for Emerald.) 